JOURNAL OF

SYMPLECTIC GEOMETRY

Volume 9, Number 2, 147-160, 2011

\title{
NEGATIVE INFLATION AND STABILITY IN SYMPLECTOMORPHISM GROUPS OF RULED SURFACES
}

\author{
Olguţa Buşe
}

\begin{abstract}
Consider symplectic ruled surfaces $M_{\lambda}^{g}=\left(\Sigma_{g} \times S^{2}, \lambda \sigma_{\Sigma_{g}} \oplus \sigma_{S^{2}}\right)$ such that $\Sigma_{g}$ has area $\lambda$ and $S^{2}$ has area 1 . We show that for $k \geq\lfloor g / 2\rfloor$ the homotopy type of the symplectomorphism groups $G_{\lambda}^{g}$ of $M_{\lambda}^{g}$ is constant as $\lambda$ increases in the interval $(k, k+1]$, thus generalizing an existent result of Abreu-McDuff for the rational ruled surfaces with $g=0$. We also investigate the changes in the groups $\pi_{*} G_{\lambda}^{g}$ as $\lambda$ passes an integer $k$ and show the existence of higher Samelson products in $\pi_{4 k+2 g} G_{\lambda}^{g}$ that exist only for $\lambda$ in the range $(k, k+1]$. To prove these results we introduce a refinement of the negative inflation technique introduced by $\mathrm{Li}-\mathrm{U}$ sher.
\end{abstract}

\section{Introduction and results}

The purpose of this note is to introduce and apply a refinement of the negative inflation method in a symplectic four-manifold. Inflation was first introduced by Lalonde-McDuff [13] for embedded $J$-holomorphic curves with positive self-intersection and extended later in a weaker version by Li-Usher [14] for negative self-intersection curves. Our work is based on the following:

Theorem 1.1. Fix a symplectic four-manifold $\left(M^{4}, J, \tau_{0}\right)$ such that $J$ is any $\tau_{0}$-tame almost complex structure. Assume that $M$ admits an embedded J-holomorphic curve $u:(\Sigma, j) \rightarrow\left(M^{4}, J\right)$ in a homology class $Z$ with $Z^{2}=-m$. For all $\varepsilon>0$ there exist a family of symplectic forms $\tau_{\mu}$ all taming $J$ which satisfy

$$
\left[\tau_{\mu}\right]=\left[\tau_{0}\right]+\mu a_{Z}
$$

for all $0 \leq \mu \leq \frac{\tau_{0}(Z)}{m}-\varepsilon$, where $a_{Z}$ is the Poincare dual of $Z$.

To prove it we adapt and refine McDuff's method [15] used in the case of positive curves. In the Li-Usher work, which inspired this paper, the authors prove the above result without the added tameness condition on 
our symplectic forms. They use symplectic gluing techniques and are able to show the existence of symplectic forms for a maximal range $0 \leq \mu<\frac{2 \tau_{0}(Z)}{m}$. But for $\mu>\frac{\tau_{0}(Z)}{m}$ one has $\tau_{\mu}(Z)<0$ so tameness cannot be expected for that range, hence our result is optimal under the above conditions.

While previous works exists by Gromov [11], Abreu [1], Abreu-McDuff [3], Anjos [4, 5], Abreu-Granja-Kitchloo [2], for the rational case when $g=0$, we present here most statements from [15] as we are basing our note on many of these results. In 2000, McDuff [15] studied the space of symplectomorphism groups for ruled surfaces of arbitrary genus. Recall that a topologically trivial ruled surface $M_{\lambda}^{g}$ is the total space of the topologically trivial symplectic fibration $\left(\Sigma_{g} \times S^{2}, \lambda \sigma_{\Sigma_{g}} \oplus \sigma_{S^{2}}\right) \longrightarrow\left(\Sigma_{g}, \sigma_{\Sigma_{g}}\right)$, where $\lambda>0$ and the two-forms $\sigma_{S^{2}}$ and $\sigma_{\Sigma_{g}}$ have total area 1. Accordingly, we define the symplectomorphism groups $G_{\lambda}^{g}$ by

$$
G_{\lambda}^{g}:=\operatorname{Symp}\left(\Sigma_{g} \times S^{2}, \lambda \sigma_{\Sigma_{g}} \oplus \sigma_{S^{2}}\right) \cap \operatorname{Diff}_{0}\left(M_{\lambda}^{g}\right) .
$$

The manuscript [15] exploits a homotopy fibration based on a fibration first introduced by Kronheimer [12], which in this case reads:

$$
0 \longrightarrow G_{\lambda}^{g} \longrightarrow \operatorname{Diff}_{0}\left(M_{\lambda}^{g}\right) \longrightarrow \mathcal{A}_{\lambda}^{g},
$$

where $\mathcal{A}_{\lambda}^{g}$ is the space of almost complex structures that are tamed by some form isotopic to $\lambda \sigma_{\Sigma_{g}} \oplus \sigma_{S^{2}}$. Thus one can transfer information on the topology of the spaces $\mathcal{A}_{\lambda}^{g}$ to that of the symplectomorphism groups. Let $D_{k}=A-k F \in H_{2}\left(M_{\lambda}^{g}, \mathbb{Z}\right)$, where $A$ and $F$ are the homology classes of the base and the fiber, respectively. Whenever $\lambda>k$ and $k \geq 1$ we define the sets $\mathcal{A}_{\lambda, k}^{g}$ to be the subsets of $\mathcal{A}_{\lambda}^{g}$ consisting of almost complex structures that admit irreducible $J$-holomorphic curves in the class $D_{k}$. When $g>0$ and $\lambda>0$ or $g=0$ and $\lambda \geq 1$, the main strata $\mathcal{A}_{\lambda, 0}^{g}$ of $\mathcal{A}_{\lambda}^{g}$ consist of $J$ that admit only nonnegative self-intersection curves. They provide a stratification of $\mathcal{A}_{\lambda}^{g}=\bigcup_{0 \leq k<[\lambda]} \mathcal{A}_{\lambda, k}^{g}$ as in the following proposition.

Proposition 1.1 (McDuff [15]).

(1) If $\lambda \geq 1$ when $g=0$ or $\lambda>0$ when $g>0$, we have $\mathcal{A}_{\lambda}^{g} \subset \mathcal{A}_{\lambda+\varepsilon}^{g}$ for any positive $\varepsilon$. Via (1.1) one obtains maps

$$
h_{\lambda, \lambda+\varepsilon}: G_{\lambda}^{g} \longrightarrow G_{\lambda+\varepsilon}^{g} .
$$

Moreover these maps are compatible up to homotopy under the various homotopy diagrams.

(2) For $k \geq 1$ the strata $\mathcal{A}_{\lambda, k}^{g}$ is a Frechet suborbifold of $\mathcal{A}_{\lambda}^{g}$ of codimension $4 k-2+2 g$.

(3) For any integer $k \geq 1$ the space $\mathcal{A}_{\lambda}^{0}$ is constant on a $(k, k+1]$. Moreover if $0<\varepsilon \leq 1$ one has $\mathcal{A}_{k+\varepsilon}^{0} \backslash \mathcal{A}_{k}^{0}=\mathcal{A}_{k+\varepsilon, k}^{0}$. 
We will obtain a generalization of item (3) above for the higher genus cases. McDuff's proof of item (3) is based on the positive inflation technique. To be able to do that, she needs the existence of appropriate embedded positive curves for every $J$. While suitably nontrivial Gromov invariants provide such curves for regular almost complex structures, they only translate to the existence of curves with singularities for the nongeneric $J$. Finding sufficient embedded curves for such nongeneric $J$ requires a detailed study of all the possible singularities and multiple cover curves, which led to only limited success in the attempt to generalize point (3) to all $g>0$. Our method allows us to circumvent this analysis as the embedded negative curves will be present in these strata by their very definition. Hence we can use negative inflation to effortlessly prove the following result.

Proposition 1.2. If $g>0$ we have $\mathcal{A}_{\lambda, k}^{g}=\mathcal{A}_{k+\varepsilon, k}^{g}$ whenever $k>0$, any $\lambda>k$ and any $\varepsilon>0$.

The following result from [15] describes the known ranges of stability regarding the main stratum $\mathcal{A}_{\lambda, 0}^{g}$.

Proposition 1.3 (McDuff [15]). For any $g \geq 1$ the strata $\mathcal{A}_{\lambda, 0}^{g}$ is constant for all $\lambda>\lfloor g / 2\rfloor$. In particular for $g=1$ we have that $\mathcal{A}_{\lambda, 0}^{1}$ is constant for any positive $\lambda$.

Combining the results from Propositions 1.2 and 1.3 we obtain

Proposition 1.4. $\mathcal{A}_{k+\varepsilon}^{g} \backslash \mathcal{A}_{k}^{g}=\mathcal{A}_{k+\varepsilon, k}^{g}$ and $\mathcal{A}_{\lambda}^{g}$ is constant on the interval $0<\varepsilon \leq 1$ for all $k \geq\lfloor g / 2\rfloor$.

This allows us to improve the results regarding the higher genus case. In particular we obtain the following stability result:

Theorem 1.2. The homotopy type of $G_{\lambda}^{g}$ is constant for $k<\lambda \leq k+1$, for any integer $k \geq\lfloor g / 2\rfloor$. Moreover as $\lambda$ passes the integer $k+1$ all the groups $\pi_{i}, i=0, \ldots, 4 k+2 g-1$ do not change.

1.1. Asymptotic results. McDuff showed that the inclusions $i: G_{\lambda}^{g} \longrightarrow$ $\operatorname{Diff}_{0}\left(M^{g}\right)$ lift to maps $\tilde{i}: G_{\lambda}^{g} \longrightarrow \mathcal{D}_{0}^{g}$ where $\mathcal{D}_{0}^{g}$ is the identity component of the subgroup of diffeomorphisms that preserve the $S^{2}$ fibers. The following proposition gives the homotopy limit of the groups $G_{\lambda}^{g}$ :

Proposition 1.5 (McDuff $[\mathbf{1 5}]$ ). The homotopy limit $G_{\infty}^{g}:=\lim _{\lambda \longrightarrow \infty} G_{\lambda}^{g}$ exists and it is homotopic to $\mathcal{D}_{0}^{g}$. 
The rational homotopy type of $\mathcal{D}_{0}^{g}$ is well understood in [15], as we can see in the following proposition:

Proposition 1.6 (McDuff $[\mathbf{1 5}])$. For $g>0$ the vector spaces $\pi_{i}\left(\mathcal{D}_{0}^{g}\right) \otimes \mathbb{Q}$ are described by the following results:

$$
\begin{array}{lll}
\operatorname{dim} \pi_{0}\left(\mathcal{D}_{0}^{g}\right) \otimes \mathbb{Q}=0 & \text { for all } g>0 & \\
\operatorname{dim} \pi_{1}\left(\mathcal{D}_{0}^{g}\right) \otimes \mathbb{Q}=1 & \text { for all } g>1 & \\
\operatorname{dim} \pi_{1}\left(\mathcal{D}_{0}^{g}\right) \otimes \mathbb{Q}=3 & \text { for } g=1 \\
\operatorname{dim} \pi_{2}\left(\mathcal{D}_{0}^{g}\right) \otimes \mathbb{Q}=2 g & \text { for all } g>0 \\
\operatorname{dim} \pi_{3}\left(\mathcal{D}_{0}^{g}\right) \otimes \mathbb{Q}=1 & \text { for all } g>0 & \\
\operatorname{dim} \pi_{i}\left(\mathcal{D}_{0}^{g}\right) \otimes \mathbb{Q}=0 & \text { for all } g>0, \quad i>3 .
\end{array}
$$

Moreover, based on the partial analysis of the strata of the spaces of almost complex structure $\mathcal{A}_{\lambda}^{g}$, McDuff showed that the map $\tilde{i}: G_{\lambda}^{g} \longrightarrow \mathcal{D}_{0}^{g}$ yields a surjective map on all the rational homotopy groups for all genus $g>0$ and all $\lambda>0$. Theorem 1.2 allows us to improve this result to an isomorphism for a suitable range of $\lambda$.

Proposition 1.7. There exist maps $\tilde{i}: G_{\lambda}^{g} \longrightarrow \mathcal{D}_{0}^{g}$ that induce a surjection on all rational homotopy groups for all $g>0$ and $\lambda>0$. If we take $k \geq\lfloor g / 2\rfloor$ and we restrict to the range $k<\lambda \leq k+1$ the maps $\tilde{i}$ induce isomorphisms $\tilde{i}_{*}: \pi_{j} G_{\lambda}^{g} \longrightarrow \pi_{j} \mathcal{D}_{0}^{g}$ for all $j=0, \ldots, 4 k+2 g-1$.

Therefore when we look at the stability ranges $\lfloor g / 2\rfloor \leq k<\lambda \leq k+1$ these results allow us to completely understand the rational homotopy groups $\pi_{j} G_{\lambda}^{g} \otimes \mathbb{Q}$ for all $3<j<4 k+2 g$. Any nontrivial element in $\pi_{4 k+2 g} G_{\lambda}^{g} \otimes \mathbb{Q}$ must vanish as $\lambda$ passes the integer $k+1$. We call such elements that do not survive in the homotopy limit fragile. Combining the results of Propositions 1.7 and 2.12 from McDuff [15] one can easily obtain a fragile element. Instead, we will find such nontrivial elements by using the above results and the techniques developed in Buse [9]. This allow us to exhibit fragile elements that live in the $\lambda$-range between two consecutive integers that have the extra feature of being higher Samelson products of certain nontrivial loops $\gamma^{g}$ in $\pi_{1} G_{\lambda}^{g}$.

This generalize the existence of a regular Samelson product $[\gamma, \gamma] \in \pi_{2} G_{1}^{1}$ found by Buse $[\mathbf{9}]$ for the torus case when $g=1$.

Proposition 1.8. For all genus $g \geq 2$ and all $k \geq\lfloor g / 2\rfloor$, for $k \leq \lambda<k+1$ there exist $a \gamma^{g} \in \pi_{1} G_{\lambda}^{g} \otimes \mathbb{Q}$ with nonvanishing Samelson product of order $r=2 k+g+1,0 \neq w_{k}^{g} \in S^{(r)}\left(\gamma^{g}\right) \subset \pi_{4 k+2 g} G_{\lambda}^{g} \otimes \mathbb{Q}$.

If we put together the results from Propositions 1.6 to 1.8 we obtain the following partial characterization of the rational homotopy groups $\pi_{*} G_{\lambda}^{g} \otimes \mathbb{Q}$ : 
Proposition 1.9. For $g>0, k \geq\lfloor g / 2\rfloor$ any positive integer, and for all $\lambda$ satisfying $k<\lambda \leq k+1$, the vector space $\pi_{*} G_{\lambda}^{g} \otimes \mathbb{Q}$ are described by the following results:

$$
\begin{aligned}
& \operatorname{dim} \pi_{0} G_{\lambda}^{g} \otimes \mathbb{Q}=0 \quad \text { for all } g>0 \\
& \operatorname{dim} \pi_{1} G_{\lambda}^{g} \otimes \mathbb{Q}=1 \quad \text { for all } g>1 \\
& \operatorname{dim} \pi_{1} G_{\lambda}^{g} \otimes \mathbb{Q}=3 \quad \text { for } g=1 \\
& \operatorname{dim} \pi_{2} G_{\lambda}^{g} \otimes \mathbb{Q}=2 g \text { for all } g>0 \\
& \operatorname{dim} \pi_{3} G_{\lambda}^{g} \otimes \mathbb{Q}=1 \quad \text { for all } g>0 \\
& \operatorname{dim} \pi_{i} G_{\lambda}^{g} \otimes \mathbb{Q}=0 \quad \text { for all } g>0, \quad 3<i<4 k+2 g \\
& \operatorname{dim} \pi_{4 k+2 g} G_{\lambda}^{g} \otimes \mathbb{Q} \geq 1 \text {. }
\end{aligned}
$$

1.2. Further questions. Our stability results open the way to explore the complete rational homotopy type of the groups $G_{\lambda}^{g}$, for the provided stability ranges with $\lambda \geq\lfloor g / 2\rfloor$, thus extending the work of Abreu-McDuff [3] done in the genus zero cases to the higher genus. This entails understanding the dimension of the groups $\operatorname{dim} \pi_{j} G_{\lambda}^{g} \otimes \mathbb{Q}, j \geq 4 k+2 g$, or, equivalently, how many different fragile elements exist. This requires different methods that study the topology of the links of the new strata $\mathcal{A}_{k+\varepsilon, k}^{g}$ to the remaining subset of $\mathcal{A}_{k+\varepsilon}^{g}$.

In a different direction, one would like to understand the behavior of the main strata $\mathcal{A}_{\lambda, 0}^{g}$ on the ranges $0<\lambda \leq\lfloor g / 2\rfloor$, thus settling possible stability results for the remaining $\lambda$ ranges $0<\lambda \leq\lfloor g / 2\rfloor$. These ideas will be pursued by the author in a different paper.

\section{Proof of the negative inflation theorem}

Proof of Theorem 1.1. The proof largely follows the lines of McDuff's proof of Lemma 3.1 in [15]. We need to take special care to bound away from zero the function $a$ defined below in (2.5), as it is no longer greater than 1 as in the positive inflation case.

Take $\mathcal{N}(Z)$ a neighborhood of $Z$ consisting of the unit disk bundle of a complex line bundle $\mathcal{L}$ over the curve in class $Z$. Denote by $r$ the radial coordinate. We renormalize $\tau_{0}$ such that $\tau_{0}(Z)=1$. Denote by $\sigma_{Z}$ the area form on $Z$ such that $\int_{Z} \sigma_{Z}=1$. We then can choose a connection on $\mathcal{L}$ such that the connection one-form $\alpha$ obeys $d \alpha=m \pi^{*}\left(\sigma_{Z}\right)$ where $\pi$ is the bundle projection, and such that $\int_{Z} \sigma_{Z}=1$.

We use the symplectic neighborhood theorem to get, via isotopy, that, for a sufficiently small $r \leq r_{0}$, the symplectic form $\tau_{0}$ is isotopic to the following form:

$$
\tau_{0} \approx \pi^{*}\left(\sigma_{Z}\right)+d\left(r^{2} \alpha\right)=\left(1+m r^{2}\right) \pi^{*} \sigma_{Z}+2 r d r \wedge \alpha .
$$


For all $\mu \in\left(0, \frac{1}{m}-\varepsilon\right]$ we will take $\tau_{\mu}$ to be of the form:

$$
\begin{aligned}
\tau_{\mu} & =\pi^{*} \sigma_{Z}+d\left(r^{2} \alpha\right)-d\left(f_{\mu}(r) \alpha\right) \\
& =\left(1+m r^{2}-m f_{\mu}\right) \pi^{*} \sigma_{Z}+\left(2 r-f_{\mu}^{\prime}\right) d r \wedge \alpha .
\end{aligned}
$$

For each $\mu$, the functions $f_{\mu}(r)$ will be nonincreasing positive functions of $r$ supported in a neighborhood $r \leq r_{0}$, constant in a smaller neighborhood near $r=0$ such that $f_{\mu}(0)=M<\frac{1}{m}-\varepsilon$. Note that with this choice the cohomology class $\left[\tau_{\mu}\right]=\left[\tau_{0}\right]+f(0) a_{Z}$ and it satisfies the inequality in the statement of the theorem for the normalized $\tau_{0}$. It remains for us to choose $f_{\mu}$ such that $\tau_{\mu}$ tames the given $J$.

At each point $p \in \mathcal{N}(Z)$, consider a splitting of tangent space $T_{p} \mathcal{N}(Z)$ into $E_{H} \oplus E_{F}$, where $E_{H} \subset \operatorname{Ker} d r \cap \operatorname{Ker} \alpha$ and $E_{F}$ is tangent to the fiber of $\mathcal{L}$. Note that since the curve at $r=0$ is $J$-holomorphic we can assume that the fibers of $\mathcal{L}$ are $J$-holomorphic at $r=0$. By the way we have chosen $\tau_{\mu}$ the spaces $E_{H}$ and $E_{F}$ are orthogonal with respect to $\tau_{\mu}$ and $\tau_{0}$. Then we can pick framings of the bundle $(u, v) \in E_{H} \oplus E_{F}$ so that the following holds:

$$
\tau_{0}\left((u, v),\left(u^{\prime}, v^{\prime}\right)\right)=u^{T} J_{0}^{T} u^{\prime}+v^{T} J_{0}^{T} v^{\prime},
$$

where $J_{0}$ is the standard matrix $J_{0}=\left(\begin{array}{cc}0 & -1 \\ 1 & 0\end{array}\right)$. Moreover,

$$
\begin{aligned}
\tau_{\mu}\left((u, v),\left(u^{\prime}, v^{\prime}\right)\right)= & \left(\left(1+m r^{2}-m f_{\mu}\right) \pi^{*} \sigma_{Z}\right. \\
& \left.\left.+\left(2 r-f_{\mu}^{\prime}\right) d r \wedge \alpha\right)\right)\left((u, v),\left(u^{\prime}, v^{\prime}\right)\right) \\
= & \left(1+m r^{2}-m f_{\mu}\right) \frac{1+m r^{2}}{1+m r^{2}} \pi^{*} \sigma_{Z} \\
& \left.+\left(2 r-f_{\mu}^{\prime}\right) \frac{2 r}{2 r} d r \wedge d \alpha\right]\left((u, v),\left(u^{\prime}, v^{\prime}\right)\right) \\
= & \frac{1+m r^{2}-m f_{\mu}}{1+m r^{2}}\left(1+m r^{2}\right) \pi^{*} \sigma_{Z}\left((u, v),\left(u^{\prime}, v^{\prime}\right)\right) \\
& +\frac{2 r-f_{\mu}^{\prime}}{2 r} 2 r d r \wedge d \alpha\left((u, v),\left(u^{\prime}, v^{\prime}\right)\right) \\
= & a u^{T} J_{0}^{T} u^{\prime}+b v^{T} J_{0}^{T} v^{\prime},
\end{aligned}
$$

where for each $\mu$ we define the functions $a(r)$ and $b(r)$ by

$$
a:=1-\frac{m f_{\mu}}{1+m r^{2}} \quad b:=1-\frac{f_{\mu}^{\prime}}{2 r} .
$$

Clearly $b \geq 1$, and $a$ is bounded away from zero. In fact $\inf _{\left\{r \leq r_{0}\right\}} a(r)$ is a strictly positive number, which is decreasing and converging to 0 as the parameter $\varepsilon$ converges to zero.

Using the chosen framings of the bundle we can write $J_{p}=\left(\begin{array}{ll}A & B \\ C & D\end{array}\right)$. We need to prove that $\tau_{\mu}$ tames $J$, which in coordinates is equivalent to 
$\tau_{\mu}\left((v, w), J_{p}(v, w)\right)>0$ or

$$
a v^{T} J_{0}^{T} A v+a v^{T} J_{0}^{T} B w+b w^{T} J_{0}^{T} C v+b w^{T} J_{0}^{T} D w>0
$$

for all $(v, w)$ in $E_{H} \oplus E_{F}$.

But since $v$ is $J$-holomorphic $B=C=0$ when $r=0$. Since we know that $\tau_{0}$ tames $J$ this translates into

$$
\tau_{0}\left((v, w), J_{p}(v, w)\right)=a v^{T} J_{0}^{T} A v+b w^{T} J_{0}^{T} D w>0
$$

whenever $(v, w) \neq(0,0)$.

Given that $B=C=0$ and $J_{p}^{2}=\left(\begin{array}{cc}A & 0 \\ 0 & D\end{array}\right)^{2}=-I d$ when $r=0$, we can find a neighborhood $r \leq r_{0}$ and some positive constants $c, c^{\prime}$ depending only on $J$ so that

$$
\|v\|^{2} \leq c v^{T} J_{0}^{T} A v, \quad\|w\|^{2} \leq c w^{T} J_{0}^{T} D w
$$

and

$$
\left\|v^{T} J_{0}^{T} B w\right\| \leq c^{\prime} r\left(\|v\|^{2}+\|w\|^{2}\right), \quad\left\|w^{T} J_{0}^{T} C v\right\| \leq c^{\prime} r\left(\|v\|^{2}+\|w\|^{2}\right),
$$

for all $p$ in the given $r$-neighborhood and all $(v, w) \in T_{p} \mathcal{N}(Z)$. Recall that $0<\inf _{\left\{r \leq r_{0}\right\}} a(r)<1$. We will replace $c^{\prime}$ with the larger number

$$
c^{\prime \prime}=\frac{c^{\prime}}{\inf _{\left\{r \leq r_{0}\right\}} a(r)} .
$$

Then the inequalities (2.9) yield

$$
\left\|v^{T} J_{0}^{T} B w\right\| \leq c^{\prime \prime} r\left(\|v\|^{2}+\|w\|^{2}\right), \quad\left\|w^{T} J_{0}^{T} C v\right\| \leq c^{\prime \prime} r\left(a\|v\|^{2}+\|w\|^{2}\right) .
$$

Using inequalities (2.8) and (2.10) we obtain

$$
\begin{aligned}
\left\|a v^{T} J_{0}^{T} B w\right\|+\left\|b w^{T} J_{0}^{T} C v\right\|= & \sqrt{a}\left\|\sqrt{a} v^{T} J_{0}^{T} B w\right\|+\sqrt{b}\left\|\sqrt{b} w^{T} J_{0}^{T} C v\right\| \\
\leq & c^{\prime \prime} r \sqrt{a}\left(\sqrt{a}^{2}\|v\|^{2}+\|w\|^{2}\right) \\
& +c^{\prime \prime} r \sqrt{b}\left(a\|v\|^{2}+\sqrt{b}^{2}\|w\|^{2}\right) \\
\leq & c^{\prime \prime} r(\sqrt{a}+\sqrt{b})\left(a\|v\|^{2}+b\|w\|^{2}\right) \\
\leq & c c^{\prime \prime} r(\sqrt{a}+\sqrt{b})\left(a v^{T} J_{0}^{T} A v+b w^{T} J_{0}^{T} D w\right),
\end{aligned}
$$

where the last inequality uses (2.8).

We need to find an appropriate small $r_{1}$ such that for $r<r_{1}$ and for any $\mu<\frac{1}{m}-\varepsilon$ we can find functions $f_{\mu}$ so that the expression $K:=c c^{\prime \prime} r(\sqrt{a}+$ $\sqrt{b})<1$. We will choose $f_{\mu}$ so that $-f_{\mu}^{\prime}<c^{\prime \prime \prime} / r$ for $c^{\prime \prime \prime}$ an appropriate constant. Note that for any choice of $r_{1}$ and small $c^{\prime \prime \prime}$ as in the relation 
below, we can truncate and smooth out $f_{\mu}=$ const $-c^{\prime \prime \prime} \log r$ so that $f_{\mu}(0)=$ $M<\frac{1}{m}-\varepsilon$, and $f_{\mu}\left(r_{1}\right)=0$.

First we pick an $r^{\prime}$ so that for $r<r^{\prime}$ the inequality $c c^{\prime \prime} r \sqrt{a} \leq c c^{\prime \prime} r \leq \frac{1}{2}$ holds.

We will further restrict the $r$-neighborhood by picking a small $r_{1}<r^{\prime}$ and a small $c^{\prime \prime \prime}$ so that the following sequence on inequalities hold:

$$
c c^{\prime \prime} r \sqrt{b} \leq c c^{\prime \prime} r \sqrt{1-\frac{f_{\mu}^{\prime}}{2 r}} \leq c c^{\prime \prime} \sqrt{r^{2}+c^{\prime \prime \prime}}<\frac{1}{2} .
$$

For such choices of $r_{1}, c^{\prime \prime \prime}$ and $f_{\mu}$ with $-f_{\mu}^{\prime} \leq c^{\prime \prime \prime} / r$ as above we obtain that $K=c c^{\prime \prime} r(\sqrt{a}+\sqrt{b})<1$ for all $r<r_{1}$.

Then the tameness relation (2.6) follows from (2.11) because in general if $\beta+\gamma+\delta+\eta$ with $\beta>0$ and $\gamma>0$ and $|\delta|+|\eta| \leq K(\beta+\gamma)$, then whenever $\frac{1}{K}>1$ we have

$$
\beta+\gamma+\delta+\eta>\frac{1}{K}(|\delta|+|\eta|)+\delta+\eta \geq 0
$$

\section{Consequences for the symplectomorphism groups of $M_{\lambda}^{g}$}

If we denote by $\mathcal{S}_{\lambda}^{g}$ the space of all symplectic forms strongly isotopic to $\lambda \sigma_{\Sigma_{g}} \oplus \sigma_{S^{2}}$, Moser's argument gives the following fibration:

$$
0 \longrightarrow G_{\lambda}^{g} \longrightarrow \operatorname{Diff}_{0}\left(M_{\lambda}^{g}\right) \longrightarrow \mathcal{S}_{\lambda}^{g}
$$

McDuff showed the spaces $\mathcal{A}_{\lambda}^{g}$ and $\mathcal{S}_{\lambda}^{g}$ are homotopy equivalent and thus obtained the homotopy fibration (1.1). These fibrations hold in general for any compact symplectic manifold; but to show that the inclusions $\mathcal{A}_{\lambda}^{g} \subset$ $\mathcal{A}_{\lambda+\varepsilon}^{g}$ one uses a very specific feature of symplectic ruled surfaces; namely, as is shown for instance in [15] Lemma 4.1, for each $J \in A_{\lambda}^{g}$, the manifold $M_{\lambda}^{g}$ admits a smooth foliation by $J$-holomorphic spheres in the fiber class $F$. Moreover, the success in stratifying these spaces by strata $\mathcal{A}_{\lambda, k}^{g}$ of finite codimension is also very specific to the structure of the ruled surfaces. It uses both the foliation above to show that any $J$-holomorphic curve $u$ in the class $A-k F$ must be embedded(see below), and good general regularity results from Hofer-Lizan-Sikorav [10] regarding the dimension of the cokernel of the linearized Cauchy-Riemann operator $D u$. But arguably the most important feature of the symplectic ruled surfaces that allows us to draw conclusion on stability results in the spaces $\mathcal{A}_{\lambda}^{g}$ is that they have a lot of embedded curves featured via nontrivial Gromov invariants. These arguments are for instance essential in McDuff's proof for the stability of the main strata $\mathcal{A}_{\lambda, 0}^{g}$, $\lambda>\lfloor g / 2\rfloor$.

As we explained in the introduction, our contribution to improving these arguments is to show the stability of all the other strata $\mathcal{A}_{\lambda, k}^{g}$. 
Although the abundance of $J$-holomorphic curves is inherently used by us, as negative inflation always requires embedded curves, we will no longer rely on nontrivial Gromov invariants.

Proof of Proposition 1.2. Let $J \in \mathcal{A}_{\lambda, k}^{g}$. Since the symplectic form $\omega_{\lambda}$ evaluates positively on a $J$-holomorphic curve in class $D_{k}$, we must have $\lambda>k$.

By the definition of the strata $\mathcal{A}_{\lambda, k}^{g}$ there must be an irreducible $J$-holomorphic curve in class $D_{k}$. This curve must be in fact embedded. To see this, note that since for each $J \in A_{\lambda}^{g}$, the manifold $M_{\lambda}^{g}$ admits a smooth foliation by $J$-holomorphic spheres in the fiber class $F$, and $(A-k F) \cdot F=1$. If $u$ had a singularity, the multiplicity of the intersection between the fiber $F$ passing through that point and the curve would be strictly greater than one, and thus positivity of intersection would contradict the fact that $(A-k F) \cdot F=1$. Hence the curve $u$ must either intersect any $J$-holomorphic fiber transversely at a smooth point $u$ is an embedding.

Then we apply Theorem 1.1 and get a family of symplectic forms $\tau_{\mu}$, all taming $J$, whose cohomology classes are

$$
\left.\left[\tau_{\mu}\right]=\left[\lambda \sigma_{\Sigma_{g}} \oplus \sigma_{S^{2}}\right]+\mu P D(A-k F)\right],
$$

where $0<\mu \leq \frac{\lambda-k}{2 k}-\varepsilon$. A short calculation gives us that

$$
\left[\tau_{\mu}\right]=\lambda\left[\sigma_{\Sigma_{g}}\right]+\left[\sigma_{S^{2}}\right]+\mu\left(\left[\sigma_{S^{2}}\right]-k\left[\sigma_{\Sigma_{g}}\right]\right)=(\lambda-\mu k)\left[\sigma_{\Sigma_{g}}\right]+(1+\mu)\left[\sigma_{S^{2}}\right]
$$

So if we set $\mu=\frac{\lambda-k-\delta}{2 k}$ we get that the ratio between the $\tau_{\mu}$-symplectic area of the base and that of the fiber is given by:

$$
\frac{\lambda-k(\lambda-k-\delta) / 2 k}{1+(\lambda-k-\delta) / 2 k}=\frac{k(\lambda+k+\delta)}{k+\lambda-\delta}=k+\frac{2 k \delta}{k+\lambda-\delta} .
$$

But since $\delta$ is arbitrarily small the whole expression $\frac{2 k \delta}{k+\lambda-\delta}$ can be chosen as small as we want. Hence $J \in \mathcal{A}_{k+\varepsilon^{\prime}, k}^{g}$, for any small $\varepsilon^{\prime}>0$.

The results in Theorem 1.2 are now easy to see. The first assertion follows immediately from the homotopy fibration (1.1) and Proposition 1.4. The second assertion follows from the first part of the Theorem 1.2, Proposition 1.1 part (2) and again Proposition 1.4.

The first part of Proposition 1.7 was proved in McDuff [15] Proposition 1.6 (ii). The fact that the maps $\tilde{i}_{*}: \pi_{j} G_{\lambda}^{g} \longrightarrow \pi_{j} \mathcal{D}_{0}^{g}$ with $j=0, \ldots, 4 k+$ $2 g-1$ are isomorphisms is an immediate consequence of Theorem 1.2 and Proposition 1.5.

Lastly, to obtain the results in Proposition 1.9, note that Proposition 1.7 along with the description of the diffeomorphism groups $\mathcal{D}_{0}^{g}$ from Proposition 1.6 allows one to understand all the elements in the rational homotopy groups of $G_{\lambda}^{g}$ that persist in the homotopy limit. To get all the results of Proposition 1.9 we will need to see in the next section a proof of Proposition 1.8 that describe some fragile elements in $\pi_{4 k+2 g} G_{\lambda}^{g}$, for $k<\lambda \leq k+1$. 
3.1. Fragile elements as Whitehead products. This section will be dedicated to proving Proposition 1.8.

3.1.1. Preliminaries and previous results. Following the techniques from Buse [9] we will first find higher Whitehead products in the classifying spaces $\mathrm{B} G_{\lambda}^{g}$ and get the desired higher Samelson products by desuspension to $G_{\lambda}^{g}$. Recall the following definitions introduced for example in [9].

Definition 3.1. A continuous family $F_{\lambda}: B \longrightarrow \mathrm{B} G_{\lambda}^{g}, \lambda>\lambda_{0}$ is a new family of maps with respect to $\mathrm{B} G_{\lambda_{0}}^{g}$ if it cannot be extended to a continuous family of maps $F_{\lambda}: B \longrightarrow \mathrm{B} G_{\lambda}^{g}, \lambda \geq \lambda_{0}$. Similarly, for a $\lambda>\lambda_{0}$, a nontrivial element $\left[F_{\lambda}\right] \in \pi_{*} \mathrm{~B} G_{\lambda}^{g}$ is a new element with respect to $\pi_{*} \mathrm{~B} G_{\lambda_{0}}^{g}$ if it is not the image of an element in $\pi_{*} \mathrm{~B} G_{\lambda_{0}}^{g}$ via the maps $h_{\lambda, \lambda_{0}}$ of (1.2).

We say that a nonzero element $F \in \pi_{*} \mathrm{~B} G_{\lambda_{0}}^{g}$ is fragile if it vanishes for some $\lambda>\lambda_{0}$.

Likewise, a nontrivial element $F \in \pi_{*} \mathrm{~B} G_{\lambda_{0}}^{g}$ is said to be robust if it survives in the homotopy limit $\pi_{*} \mathrm{~B} G_{\infty}^{g}$.

The same definitions apply for maps into $G_{\lambda}^{g}$.

Let us recall what the $r$ th order higher order Whitehead product $W^{(r)}(F)$ of an element $F: S^{2} \longrightarrow \mathrm{B} G_{\lambda}^{g}$ with itself represents. Consider the wedge maps, unique up to homotopy, given by

$$
g=F \vee \ldots \vee F: S^{2} \vee \ldots \vee S^{2} \longrightarrow \mathrm{B} G_{\lambda}^{g} .
$$

Denote by $T$ the codimension 2 skeleton of the $\operatorname{product}\left(S^{2}\right)^{r}$. If $i$ represents the inclusion $S^{2} \vee \ldots \vee S^{2} \longrightarrow T$, take the set of all possible extensions of $g$

$$
\mathcal{W}:=\left\{\bar{g}: T \longrightarrow \mathrm{B} G_{\lambda}^{g} \mid \bar{g} \circ i=g\right\} .
$$

The Whitehead product $W^{(r)}(F)$ is the set of elements in $\pi_{2 r-1} \mathrm{~B} G_{\lambda}^{g}$ given by the maps $\bar{g} \circ a: S^{2 r-1} \longrightarrow \mathrm{B} G_{\lambda}^{g}$, for all $\bar{g} \in \mathcal{W}$. The set $\mathcal{W}$ is nonempty if and only if all the lower Whitehead products contain the element 0 . It is immediate that this set of elements represents the obstructions to extending all possible maps $\bar{g}$ to the product $\left(S^{2}\right)^{r}$.

Those homotopy elements in Whitehead products that have infinite order can be obtained as Whitehead products in a space $\left(\mathrm{B} G_{\lambda}^{g}\right)_{\emptyset}$ called the rationalization of $\mathrm{B} G_{\lambda}^{g}$. Our Whitehead products $W^{(r)}(F)$ will be these rational Whitehead products and will consist of elements in $\pi_{2 r-1} \mathrm{~B} G_{\lambda}^{g} \otimes \mathbb{Q}$. We will refer to multiples of the map $F: \mathbb{P}^{n} \longrightarrow \mathrm{B} G_{\lambda}^{g}$ as maps given by the composites $\mathbb{P}^{n} \stackrel{h}{\longrightarrow} \mathbb{P}^{n} \stackrel{F}{\longrightarrow} \mathrm{B} G_{\lambda}^{g}$ with $h$ of arbitrary degree. It follows from the general theory $[6,7]$ that any multiple of a map $F: S^{2} \longrightarrow \mathrm{B} G_{\lambda}^{g}$ yields the same rational Whitehead products in $\pi_{2 r-1} \mathrm{~B} G_{\lambda}^{g} \otimes \mathbb{Q}$.

If we take an element $\gamma \in \pi_{*} G_{\lambda}^{g}$ and $F$ its suspension in the classifying space, the rational Samelson product $S^{(r)}(\gamma)$ is the subset of $\pi_{*} G_{\lambda}^{g} \otimes \mathbb{Q}$ 
containing desuspensions of all elements in $W^{(r)}(F)$. The following result exhibits the basic principles used in $[\mathbf{9}]$ to detect nontrivial Whitehead products.

Proposition 3.1. (Buse $[\mathbf{9}]$ ) Assume that there exists a family $F_{n+1, \varepsilon}$ : $\mathbb{P}^{n+1} \longrightarrow \mathrm{B} G_{k+\varepsilon}^{g}, \varepsilon>0$ as above satisfying

I) $F_{n+1, \varepsilon}$ and any of its multiples are new maps with respect to $\mathrm{B} G_{k}^{g}$ in the family $\mathrm{B} G_{k+\varepsilon}^{g}, \varepsilon>0$.

II) The restriction $F_{n, \varepsilon}: \mathbb{P}^{n} \longrightarrow \mathrm{B} G_{k+\varepsilon}^{g}$ is not new, i.e. it belongs to a continuous family $F_{n, \varepsilon}, \varepsilon \geq 0$.

Then at least one of the following holds

A) There is a new element with respect to $\mathrm{B} G_{k}^{g}$ in $\pi_{2 n+2}\left(\mathrm{~B} G_{k+\varepsilon}^{g}\right) \otimes \mathbb{Q}$, $\varepsilon>0$.

B) There is a nonzero element $w \in \pi_{2 n+1}\left(\mathrm{~B} G_{k}^{g}\right) \otimes \mathbb{Q}$ in the Whitehead product $W^{(n+1)}\left(F_{1}\right)$.

We should now summarize how we construct such families. To do so, let us briefly recall how several symplectic circle actions arise on $M_{\lambda}^{g}$ and how they provide robust elements in $\pi_{1} G_{\lambda}^{g}$ (see [9] Section 4.2 for a more detailed explanation).

Whenever $\lambda>k$, one can construct the symplectic manifolds $M_{\lambda}^{g}$ via symplectic reduction from disk bundles $D_{a}\left(\mathcal{O}(-2 k)_{g} \oplus \mathcal{O}_{g}\right)$ for appropriate radii $a$. The Kähler manifolds $P\left(\mathcal{O}(-2 k)_{g} \oplus \mathcal{O}_{g}\right)$, endowed with natural integrable structures $J_{k}^{g}$, admit holomorphic circle actions that act by rotating the fibers while fixing the zero section in class $A-k F$ and the section at infinity in class $A+k F$. Thus each symplectic manifold $M_{\lambda}^{g}$ is endowed with $\lfloor\lambda\rfloor$ different Hamiltonian circle actions. They give rise to the following group homomorphisms

$$
\gamma_{\lambda, k}: S^{1} \longrightarrow G_{\lambda}^{g}, \quad 1 \leq k<\lambda .
$$

These maps are essential in homotopy and moreover, based on a result of Abreu-McDuff, they all give the related elements in the rational homotopy group (see Lemma 4.3 in $[\mathbf{9}]$ )

$$
\left[\gamma_{\lambda, k}\right]=k\left[\gamma_{\lambda, 1}\right] \in \pi_{1} G_{\lambda}^{g} \otimes \mathbb{Q} .
$$

whenever $\lambda>k \geq[g / 2]$.

For ease in indexing in this section and to be consistent with the work in Buse [9] that is being used for the proofs, we will restate Proposition 1.8 for $\lambda$ between integers $(k-1, k]$. Thus we require that $k$ be always strictly greater than $\lfloor g / 2\rfloor$.

Proposition 3.2. For all genus $g \geq 1$ and all integer $k>\lfloor g / 2\rfloor$, and for $k-1 \leq \lambda<k$ there exist a $\gamma^{g} \in \pi_{1} G_{\lambda}^{g} \otimes \mathbb{Q}$ with nonvanishing Samelson product of order $r=2 k+g-1,0 \neq w_{k-1}^{g} \in S^{(r)}\left(\gamma^{g}\right) \subset \pi_{4 k+2 g-4} G_{\lambda}^{g}$. 
Proof. For each value of $k>\lfloor g / 2\rfloor$ we will apply Proposition 3.1 for a set value of $n:=2 k+g-2$.

In order to show how the hypotheses I and II of Proposition 3.1 are satisfied, let us recall the main arguments in $[\mathbf{9}]$ that allowed us to construct the family $F_{n+1, \varepsilon}: \mathbb{P}^{n+1} \longrightarrow \mathrm{B} G_{k+\varepsilon}^{g}, \varepsilon>0$. The maps $\gamma_{k+\varepsilon, k}$ described in (3.7) give rise to a family of maps

$$
H_{n+1, \varepsilon}: \mathbb{P}^{n+1} \longrightarrow \mathrm{B} G_{k+\varepsilon}^{g}, \varepsilon>0 .
$$

Based on computations of Gromov-Witten invariants, Corollary 4.5 in [9] shows that the family $H_{n+1, \varepsilon}: \mathbb{P}^{n+1} \longrightarrow \mathrm{B} G_{k+\varepsilon}^{g}, \varepsilon>0$, as well as its multiples, are new. The main idea there was to look at the symplectic fibrations associated with the maps $H_{n+1, \varepsilon}$ and show that they have nontrivial equivariant Gromov-Witten invariants $\operatorname{EGW}\left(D_{k}\right) \neq 0$. Such EGW invariants are preserved under a deformation of the symplectic structures on the fibrations, or, equivalently, under a continuous family $H_{n+1, \varepsilon}$. But a potential such map at the critical level $\varepsilon=0$ would correspond to a symplectic form $\omega_{k}$ with $\omega_{k}\left(D_{k}\right)=0$ thus could not possibly have nontrivial EGW $\left(D_{k}\right)$.

Now use a different circle action $\gamma_{k, 1}$ at the critical level when $\varepsilon=0$ to obtain from the suspension $\mathrm{B} \gamma_{k, 1}^{g}: \mathrm{B} S^{1} \longrightarrow \mathrm{B} G_{k}^{g}$ a map

$$
F_{1}: \mathbb{P}^{1} \longrightarrow \mathrm{B} G_{k}^{g} .
$$

By composition with the maps $h_{k, k+\varepsilon}$ we get a family

$$
F_{1, \varepsilon}=h_{k, k+\varepsilon} \circ F_{1}: \mathbb{P}^{1} \longrightarrow \mathrm{B} G_{k+\varepsilon}^{g}, \quad \varepsilon \geq 0 .
$$

Observe that after considering sufficient multiples for both $F_{1, \varepsilon}$ (that exists for all $\varepsilon \geq 0$ ) and $H_{n+1, \varepsilon}$ (that exists only for $\varepsilon>0$ ), we get, via the relation (3.8) that when $\varepsilon>0$ the maps $F_{1, \varepsilon}$ and $H_{1, \varepsilon}$ are homotopic to each other, have no torsion, and are homotopic to a multiple of the suspension of the circle map $\gamma_{k+\varepsilon, 1}^{g}$.

We want to build on $F_{1}$ a map $F_{n}$ whose deformations $F_{n, \varepsilon}$ obtained via composition with $h_{k, k+\varepsilon}$ are homotopic to $H_{n, \varepsilon}$. Note that the homotopy type of the map $H_{n, \varepsilon}$ is determined by all the homotopy classes of the attaching maps used to attach cells to $H_{1, \varepsilon}$.

By Proposition 1.7 the maps $h_{k, k+\varepsilon}$ induce an isomorphism between $\pi_{*} \mathrm{~B} G_{k}^{g}$ and $\pi_{*} B G_{k+\varepsilon}^{g}$ for $*<2 n$. Therefore we can pick at the critical level $\varepsilon=0$ attaching maps that are homotopic to those used when $\varepsilon>0$ to build $H_{n, \varepsilon}$ on $H_{1, \varepsilon}$. We will use such maps to build, when $\varepsilon=0$, a new map $F_{n}: \mathbb{P}^{n} \longrightarrow \mathrm{B} G_{k}^{g}$ that restricts to $F_{1}$ on the two-skeleton. Note that we can no longer extend this procedure to get a map $F_{n+1}$. Namely, not having an isomorphism between $\pi_{2 n+1} \mathrm{~B} G_{k}^{g}$ and $\pi_{2 n+1} \mathrm{~B} G_{k+\varepsilon}^{g}$ prevents us from picking an attaching map in $\pi_{2 n+1} \mathrm{~B} G_{k}^{g}$ homotopic to the one used to build $H_{n+1, \varepsilon}$ 
on $H_{n, \varepsilon}$. Evidently, by this construction, the deformations

$$
F_{n, \varepsilon}:=h_{k, k+\varepsilon} \circ F_{n}: \mathbb{P}^{n} \longrightarrow \mathrm{B} G_{k+\varepsilon}^{g}, \quad \varepsilon \geq 0 .
$$

are homotopic to $H_{n, \varepsilon}$ for $\varepsilon>0$.

Moreover, as long as $\varepsilon>0$, we can extend $F_{n, \varepsilon}$ to a map $F_{n+1, \varepsilon}$ homotopic to $H_{n+1, \varepsilon}$ by using the same attaching map as the one used to build $H_{n+1, \varepsilon}$ on $H_{n, \varepsilon}$.

Since the family $H_{n+1, \varepsilon}: \mathbb{P}^{n+1} \longrightarrow \mathrm{B} G_{k+\varepsilon}^{g}$ was new with respect to $\mathrm{B} G_{k}^{g}$, any family homotopic to it such as $F_{n+1, \varepsilon}$ must also be new with respect to $\mathrm{B} G_{k}^{g}$. Moreover this latter family has the virtue that by construction it also satisfies condition II in the hypothesis of Proposition 3.1. Since the family $F_{n+1, \varepsilon}, \varepsilon>0$ satisfies both conditions I and II of Proposition 3.1, either point A) or B) must hold. But we know that for any $\varepsilon>0$ the space $\pi_{2 n+2}\left(\mathrm{~B} G_{k+\varepsilon}^{g}\right) \otimes \mathbb{Q}=0$ hence A) cannot hold. Therefore B) must hold and we have a nonzero element $W \in \pi_{2 n+1} \mathrm{~B} G_{k}^{g} \otimes \mathbb{Q}$ that gives a nontrivial Whitehead product of order $n+1$. Recall that we defined $n=2 k+g-2$. Therefore we get an element with nonvanishing Samelson product of order $r=2 k+g-1,0 \neq w_{k-1}^{g} \in S^{(r)}\left(\gamma^{g}\right) \subset \pi_{4 k+2 g-4} G_{k}^{g}$. Since $k$ is strictly greater than $\lfloor g / 2\rfloor$ by the stability Theorem 1.2 we get an element in $\pi_{4 k+2 g-4}$ $G_{\lambda}^{g} \otimes \mathbb{Q}$ for all $k-1<\lambda \leq k$. Hence Proposition 3.2 holds.

Remark 3.1. Proposition 4.12 in $[\mathbf{9}]$ provides a weaker version of the result proved here; namely, it states the existence of nontrivial Whitehead products but only provides a range for their possible order. Instead of applying Proposition 3.1 it uses a less strong version of it, Corollary 2.7 in [9]. The key feature that allowed us to improve that result is the fact that the maps $h_{k, k+\varepsilon}$ induce isomorphism on $\pi_{*}$, for all $1 \leq *<2 n$ rather than the previously known range $1 \leq * \leq 2 g-1$.

\section{Acknowledgments}

The idea for this paper arose after attending Michael Usher's talk at IAS. The author would like to thank Dusa McDuff for very valuable suggestions and comments and a careful reading of the manuscript.

\section{References}

[1] M. Abreu, Topology of symplectomorphism groups of $S^{2} \times S^{2}$, Invent. Math. 131 (1998), 1-23.

[2] M. Abreu, G. Granja and N. Kitchloo, Compatible complex structures on symplectic rational ruled surfaces, Duke J. Math. 148(3) (2009), 539-600.

[3] M. Abreu and D. McDuff, Topology of symplectomorphism groups of rational ruled surfaces, J. Amer. Math. Soc. 13(4) (2000), 971-1009.

[4] S. Anjos, Homotopy of the symplectomorphism groups of $S^{2} \times S^{2}$, Geom. Topol., 6 (2002), 195-218. 
[5] S. Anjos and G. Granja, Homotopy decomposition of the symplectomorphism groups of $S^{2} \times S^{2}$, Topology, 43 (2004), 599-618.

[6] P. Andrews and M. Arkowitz, Sullivan's minimal model and higher order Whitehead products, Canad. J. Math. 13 (1978), 961-982.

[7] C. Allday, Rational Whitehead products and a spectral sequence of Quillen,II, Hust. J. Math. 3(3) (1977), 301-308.

[8] O. Buşe, Parametric Gromov-Witten invariants and symplectomorphism groups, Pac. J. Math. 218(2) (2005), 315-341.

[9] O. Buşe, Deformations of Whitehead products, Symplectomorphism groups, and Gromov-Witten Invariants, Int. Math. Res. Not. 215 (2010).

[10] H.Hofer, V.Lizan and J.-C. Sikorav, On the genericity for complex curves in 4-dimensional almost complex manifolds, J. Geom. Anal. 7 (1997), 149-159.

[11] M. Gromov, Pseudo holomorphic curves in symplectic manifolds, Invent. Math. 82 (1985), 307-347.

[12] P. Kronheimer, Some non-trivial families of symplectic structures, Harvard preprint.

[13] F. Lalonde and D. McDuff, The classification of ruled symplectic 4-manifolds, Math. Res. Lett. 3 (1996), 769-778.

[14] T.J. Li and M. Usher, Symplectic forms and surfaces of negative square, J. Symplectic Geom., 4(1) (2006), 71-91.

[15] D. McDuff, Symplectomorphism groups and almost complex structures, essays on geometry and related topics, Vol 1, 2, 527-556, Enseignement Math., Geneva, 2001.

[16] D. McDuff and D.A. Salamon, J-holomorphic curves and quantum cohomology, University Lecture Series 6, American Mathematical Society, Providence, RI, 1994.

[17] D. McDuff and D.A. Salamon, Introduction to Symplectic Topology, 2nd ed., Oxford University Press, 1998.

[18] G. Porter, Higher order Whitehead products, Topology 3 (1965), 123-135.

[19] G.H. Whitehead, Elements of homotopy theory, in Graduate texts in Mathematics, Springer-Verlag, 1978.

LD-270C IUPUI, 402 N. BLACKFORD St.

INDIANAPOLIS, IN 46202, USA.

E-mail address: buse@math.iupui.edu

Received 2/27/2010, accepted 10/27/2010 